Original Research

\title{
Self-reported vs RUCA rural-urban classification among North Carolina pharmacists
}

\author{
Micah E. CASTLE ${ }^{\text {Did, }}$, Casey R. TAK (D). \\ Received (first version): 20-Apr-2021 Accepted: 15-Aug-2021 Published online: 21-Aug-2021
}

\begin{abstract}
Background: The various ways in which rurality is defined can have large-scale implications on the provision of healthcare services. Objective: The purpose of this study was to identify the relationship between self-perceived urban-rural distinction and the United States (US) Census tract-based Rural-Urban Commuting Area (RUCA) scheme that defines rurality among pharmacists.

Methods: This was a secondary analysis of data collected through a web-based survey of licensed pharmacists in North Carolina. Respondents self-reported their workplace settings, zip codes, and the pharmacy services offered in their place of work. Zip codes were replaced with the corresponding RUCA codes. The relationship between self-reported classification and RUCA codes was analyzed and a chi square test was performed to measure statistical significance.

Results: Of the original survey, 584 participants reported their workplace zip code and 579 reported their workplace setting (urban, rural). A significant difference was found between pharmacists who self-reported working in rural areas and the RUCA classifications 94 (56.6\%) of the 166 participants who reported working in "rural" areas were considered "urban" according to RUCA.

Conclusions: A significant discordance between pharmacists' self-reported classification and the RUCA codes was found, with more respondents self-reporting their workplace area as "rural" as compared to the RUCA classification. Decision-makers examining the pharmacy workforce and pharmacy services should be aware of this discordance and its implications for resource allocation. We recommend the use of standardized metrics, when possible.
\end{abstract}

Keywords

Rural Population; Rural Health; Delivery of Health Care; Resource Allocation; Workplace; Pharmacists; Pharmacies; Pharmaceutical Services; Surveys and Questionnaires; North Carolina

\section{INTRODUCTION}

In 2010, data from the US census revealed that approximately 60 million Americans, or around 19 percent of the population, lived in rural areas of the United States. ${ }^{1}$ Previous literature has established that health disparities exist between urban and rural citizens; people living in rural areas are more likely to be obese, more likely to suffer from chronic diseases such as diabetes or cardiovascular diseases, less likely to be insured, and have less access to preventative care services such as cancer screenings or vaccinations. ${ }^{2-6}$ Recognizing differences in rural and urban areas helps policy makers identify which healthcare services or initiatives would be most beneficial to specific areas. For example, Talbert et al. found that while rural residents were significantly less likely to have received a pneumococcal vaccine, those who were vaccinated were more likely than their urban counterparts to have received their vaccinations from pharmacists. ${ }^{6}$ In addition, the study found that pharmacists had a significant impact on vaccination delivery in rural areas as they were more accessible than primary care providers. ${ }^{6}$

The urban-rural classification of an area is complex and varies widely according to the definition used; however, its distinction is imperative in order to better understand why disparities exist between urban and rural locations and to improve health care access. ${ }^{7}$ One standardized

Micah E. CASTLE. MPharm. School of Pharmacy, University College London, London (United Kingdom). mcastle33@gmail.com Casey R. TAK. PhD, MPH. Division of Pharmaceutical Outcomes and Policy, Eshelman School of Pharmacy, University of North Carolina at Chapel Hill, Chapel Hill, NC (United States). casey_tak@unc.edu classification system called Rural-Urban Commuting Area (RUCA) codes is a US Census tract-based classification scheme created by the Economic Research Service of the United States Department of Agriculture. These uniform RUCA codes are often used in health studies as a method to standardize the rural classification of an area based on zip codes and can ultimately influence project development for healthcare initiatives and the provision of healthcare services. ${ }^{8-11}$ The appropriate classification of rurality of an area, therefore, has large scale implications for research and resource allocation, especially in more rural areas.

While many health-related studies rely on RUCA codes to categorize rurality, RUCA categorization is problematic in that it requires study participants to disclose their zip codes, which may lead to item nonresponse. ${ }^{12}$ As there is high rate of demographic non-response in surveys, researchers may choose to omit identifiers that can be perceived as privacy violation, such as zip codes, and instead rely on self-reported demographic classifications. ${ }^{12}$ Unfortunately, self-reported classifications do not always align with standardized measurements of rurality, and the use of one over the other may alter study findings. ${ }^{13-15}$

A study done by Onega et al. demonstrated discordance between individuals' self-reported rural-urban status and RUCA categorization, while another study done by Lin et al. found significant differences between physicians' selfreported workplace geographic classifications and censusbased classification. ${ }^{11,16}$ In pharmacy, studies examining service availability and rural-urban differences have used various definitions of rurality. ${ }^{14,17-21}$ In a systematic review, Howarth et al. compared community pharmacy practice in urban and rural areas. ${ }^{14}$ In the 17 studies reviewed, six 
were from the US and used multiple definitions of rurality, including the US Census Bureau definition of $<2500$ individuals in an area, $<4000$ individuals in an area, countylevel designations, and no standard definition. For the 11 studies originating from Australia, the United Kingdom (UK), Canada, and South Africa, standardized definitions, such as the Accessibility/Remoteness Index of Australia and the UK Rural Urban Classification, were used along with population-based and non-standardized definitions. However, no current literature exists to show whether RUCA classifications are consistent with the perception of rurality among pharmacists. Given the integral role pharmacists have in the provision of healthcare in rural communities, it is critical to understand their accuracy in self-reporting geographic locations. The purpose of this study is to examine the relationship between pharmacists' self-reported geographic location and RUCA classification schemes. The knowledge generated from this study can be used to improve understanding of the impact of selfreported geographic location on healthcare services in rural versus urban settings.

\section{METHODS}

This study is a secondary analysis of previously collected data from Seamon et al., a study that focused on the pharmacists' role in access to hormonal contraception. ${ }^{22} \mathrm{~A}$ detailed description of the sampling methodology and the study sample are described elsewhere. ${ }^{22}$ Briefly, licensed pharmacists working in North Carolina were asked to complete a cross-sectional web-based survey, responding to questions that generated demographic and pharmacy practice data. The survey was administered via email in late 2018 and was open for approximately one month. A reminder email was sent a few weeks after the initial distribution. Respondents were able to self-report workplace setting and provide zip codes of these locations. The response rate was $5.9 \%$, with 713 out of 12,001 actively licensed pharmacists completing the survey. Participation in the study was voluntary and respondents were eligible to enter a raffle for the chance to win a USD25 gift card.

Of the data collected, we used a subgroup of respondents who had provided their workplace zip code. Along with zip code, pharmacists provided a self-reported definition of their workplace setting: urban, suburban, rural, and prefer not to answer. No prompts or aids were given in the survey to assist pharmacists with this determination. Other important data collected in the survey and included in this study were gender, age, pharmacy education training (Doctor of Pharmacy, Bachelor of Pharmacy, etc.), primary pharmacy workplace (community, hospital, etc.), and number of years working as a pharmacist.

To classify zip codes as urban or rural, we used the RuralUrban Commuting Area (RUCA) codes. The RUCA classifications comprise 33 distinct codes, which are generated using the population density, commuting patterns, and urbanization of an area, and every US zip code is categorized under a distinct RUCA code. ${ }^{23}$ RUCA codes were attributed to each of the reported zip codes using the updated 2019 mapping system. ${ }^{23}$ The translation of zip codes to RUCA codes resulted in 12 different RUCA classifications in our data. ${ }^{23}$ These 12 RUCA classifications were then grouped into "urban" or "non-urban" using the RUCA Classification $C$ and groupings developed by the US Federal Office of Rural Health Policy (FORHP). ${ }^{24,25}$ Classification $C$ was used as it is the most frequently used classification system to use when sorting the RUCA codes into two groups, urban and rural. $^{24}$ The additional RUCA code categorization scheme developed by the FORHP was used as a measure of robustness.

The study population was characterized with descriptive statistics, looking at frequency and percentage. Selfreported rurality was cross-tabulated with RUCA codes assigned to each zip code to examine the distribution. To align the self-reported rurality data in which respondents answered urban, suburban, or rural with the RUCA "urban" and "non-urban" codes, we dichotomized these responses and grouped "suburban" responses with the "urban" category. Respondents who chose "prefer not to answer" were excluded from analysis. The two categorizations, selfreported and RUCA were then compared with a chi squared test. A p-value of $<0.05$ was considered statistically significant. We also conducted a subgroup analysis where we examined the comparison of self-reported to RUCA among community pharmacists only. Data were analyzed in Microsoft Excel (Microsoft Corporation, Redmond, WA).

This study was reviewed and deemed exempt by The University of North Carolina at Chapel Hill Institutional Review Board

\section{RESULTS}

Of the 713 respondents, 584 provided a workplace zip code. Of the 584,379 (64.9\%) of the participants were female, 196 (33.6\%) of them had been a pharmacist for more than 20 years, 314 (53.8\%) were 39 years old or younger, $353(60.5 \%)$ of them went to a North Carolina pharmacy school, and 429 (73.5\%) of them had a Doctor of Pharmacy (PharmD). Most participants in this study indicated they worked in a community pharmacy (315 [53.9\%]), with the majority in a chain community pharmacy (213 [36.5\%]) and fewer in an independent community pharmacy (99 [17.0\%]). Additional demographic details are located in Table 1.

Altogether, 579 (99.1\%) out of 584 participants selfreported the geographic location of their workplace. Of the total participants, 178 (30.5\%) reported working in an urban area, 235 (40.2\%) reported suburban, and 166 (28.4\%) reported rural. Four participants $(0.68 \%)$ did not respond to the question. Table 2 shows the breakdown of self-reported responses by RUCA code. The most common RUCA codes were 1 - Metropolitan Core $(n=435 ; 74.5 \%), 4$ - Micropolitan Core $(n=66 ; 11.3 \%)$, and 2 - High Commuting to a Metropolitan ( $n=43 ; 7.4 \%)$. RUCA Categorization C identified $483(83.4 \%)$ individuals as working in an urban area and 96 (16.6\%) individuals working in rural areas; the FORHP categorized 479 (82.7\%) participants as working in urban areas and 100 (17.3\%) participants working in rural areas (see Table 3 for complete results). The differences between the selfreported data and both the RUCA and FORHP classifications were found to be statistically significant $(p<0.05)$. 


\begin{tabular}{|c|c|}
\hline Variable & $\mathrm{N}(\%)$ \\
\hline \multicolumn{2}{|l|}{ Gender } \\
\hline \multirow{3}{*}{$\begin{array}{r}\text { Male } \\
\text { Female } \\
\text { Did not/prefer not to answer }\end{array}$} & $201(34.42)$ \\
\hline & $379(64.90)$ \\
\hline & $4(0.68)$ \\
\hline \multicolumn{2}{|l|}{ Years Pharmacist } \\
\hline Less than 5 years & $204(34.93)$ \\
\hline 6-10 years & $82(14.04)$ \\
\hline $11-20$ years & $102(17.47)$ \\
\hline More than 20 years & $196(33.56)$ \\
\hline \multicolumn{2}{|l|}{ Primary Practice } \\
\hline Community Practice - chain & $213(36.47)$ \\
\hline Community Practice - independent & $99(16.95)$ \\
\hline Community pharmacy owner & $3(0.51)$ \\
\hline Staff hospital pharmacist & $39(6.68)$ \\
\hline Clinical pharmacist - hospital & $58(9.93)$ \\
\hline Clinical pharmacist - ambulatory care & $45(7.71)$ \\
\hline Academia & $8(1.37)$ \\
\hline Managed care pharmacy & $11(1.88)$ \\
\hline Long-term care pharmacy & $15(2.57)$ \\
\hline Mail order pharmacy & $2(0.34)$ \\
\hline Industry & $18(3.08)$ \\
\hline Hospital pharmacy administration & $20(3.42)$ \\
\hline Other & $51(8.73)$ \\
\hline Did not answer & $2(0.34)$ \\
\hline \multicolumn{2}{|l|}{ Age } \\
\hline Younger than 39 years old & $314(53.77)$ \\
\hline $40-59$ years old & $179(30.65)$ \\
\hline 60 years or older & $87(14.90)$ \\
\hline Did not/prefer not to answer & $4(0.68)$ \\
\hline \multicolumn{2}{|l|}{ School of pharmacy (SOP) } \\
\hline Non-North Carolina SOP & $224(38.36)$ \\
\hline North Carolina SOP & $353(60.45)$ \\
\hline Did not answer & $7(1.20)$ \\
\hline \multicolumn{2}{|l|}{ *Higher Education } \\
\hline PharmD & $429(73.46)$ \\
\hline BPharm & $217(37.16)$ \\
\hline Pharmacy residency & $97(16.61)$ \\
\hline Fellowship & $11(1.88)$ \\
\hline $\begin{array}{l}\text { *Participants were able to select more thar } \\
\text { education category when filling out surve } \\
\text { residency includes both PGY1 and PGY2 }\end{array}$ & $\begin{array}{l}\text { one higher } \\
\text { Pharmacy }\end{array}$ \\
\hline
\end{tabular}

In the stratified analysis comparing the self-reported geographic location to RUCA Categorization $\mathrm{C}$ of community and non-community pharmacists separately, we found that the majority of responses from both groups of pharmacists who self-reported working in urban or suburban areas did corresponded to the RUCA categorization of the area - of the 315 community pharmacists, $212(67.3 \%)$ reported urban or suburban compared to 193 (61.3\%) as classified by RUCA. In contrast, $102(32.4 \%)$ respondents self-reported working in a rural area compared to $47(14.9 \%)$ considered rural according to RUCA codes. Only 47 (46.1\%) respondents who reported their workplace location as rural aligned with RUCA classifications. Similar findings were found among non- community pharmacists.

\section{DISCUSSION}

The data generated from this study suggest self-reported rural-urban classifications among pharmacists working in North Carolina do not always align with zip code-based RUCA categorizations. Of the participants who reported their zip codes, $20 \%$ of them identified the rurality of their workplace geographic location inconsistently with RUCA categorizations (both $\mathrm{C}$ and FORHP). Interestingly, it was more likely for respondents to categorize their location as more rural rather than more urban. This study also looked specifically at the responses of community pharmacists, as they are embedded in their communities and often the subject of scientific inquiry. As with all pharmacists, they were more likely to perceive their working location as more rural rather than more urban. Over half of the community pharmacists identified their location as rural when it was categorized as urban according to RUCA codes compared to fewer than $10 \%$ of respondents self-reporting their location as rural when it was categorized as urban.

The findings of this study are consistent with the limited studies that have compared perception of rural-urban areas with US Census rurality definitions. Onega et al. found a significant discordance between self-reported data and RUCA classifications among a general adult population in New Hampshire and Vermont. ${ }^{16}$ As with our study, both urban and rural residents were likely to perceive their community differently from its classification, either less urban for those in urban areas or more urban for those in rural areas. This discordant attribution may be due the perception of individuals toward the environment, resources and structure of their communities. ${ }^{16}$ A 1997 study done by Lin et al. found that physicians also inconsistently self-identified their practice locations compared to census-defined urban-rural classification. ${ }^{11}$ Additionally, this study identified that many differences in workplace practices seen between rural and urban populations depend on the classification system used to categorize rurality. ${ }^{11}$ Our study contributes to the conclusions of these previous studies as it further identifies discordance in rurality classifications and provides novel information on rurality perception among pharmacists. These data are particularly important in the implications of these collective findings as pharmacists are an essential healthcare professional, arguably more so in rural areas.

Interpretation of healthcare research is often based on the understanding of an area's urban-rural context, as demonstrated in well-reported instances of differences in health behaviors and prevalence of disease between rural and urban communities., ${ }^{3,8,9}$ Conflicting rurality classifications, however, can influence or alter the findings of these studies, as conclusions are based on the mode of

\begin{tabular}{|c|c|c|c|c|c|c|c|c|c|c|c|c|c|}
\hline \multirow{2}{*}{ Self-reported classification } & \multicolumn{13}{|c|}{ RUCA classifications $^{23}$} \\
\hline & 1 & 2 & 2.1 & 3 & 4 & 4.1 & 5 & 6 & 7 & 7.1 & 8 & 10 & Total \\
\hline Urban & 169 & 4 & & 1 & 4 & & & & & & & & 178 \\
\hline Suburban & 207 & 8 & & & 14 & & 1 & & 4 & & & 1 & 235 \\
\hline Rural & 55 & 30 & 1 & 4 & 48 & 2 & 4 & 1 & 9 & 2 & 1 & 9 & 166 \\
\hline Prefer not to answer & 4 & 1 & & & & & & & & & & & 5 \\
\hline Total & 435 & 43 & 1 & 5 & 66 & 2 & 5 & 1 & 13 & 2 & 1 & 10 & 584 \\
\hline
\end{tabular}




\begin{tabular}{|c|c|c|c|c|c|c|}
\hline \multirow[b]{2}{*}{ Self-reported } & \multicolumn{3}{|c|}{ RUCA C } & \multicolumn{3}{|c|}{ FORHP } \\
\hline & $\begin{array}{c}\text { Urban } \\
\mathrm{N},(\%)^{* *}\end{array}$ & $\begin{array}{c}\text { Rural } \\
\mathrm{N},(\%)^{* *}\end{array}$ & Total & $\begin{array}{c}\text { Urban } \\
\mathrm{N},(\%)^{* *}\end{array}$ & $\begin{array}{c}\text { Rural } \\
\mathrm{N},(\%)^{* *}\end{array}$ & Total \\
\hline Urban & 389 (94.2\%) & $24(5.8 \%)$ & 413 & 389 (94.2\%) & $24(5.8 \%)$ & 413 \\
\hline Rural & 94 (56.6\%) & $72(43.4 \%)$ & 166 & $90(54.2 \%)$ & 76 (45.8\%) & 166 \\
\hline \multirow[t]{2}{*}{ Total } & 483 & 96 & $579 *$ & 479 & 100 & 579 \\
\hline & \multicolumn{3}{|c|}{$p<0.0001$} & \multicolumn{3}{|c|}{$p<0.0001$} \\
\hline
\end{tabular}

urban-rural categorization. ${ }^{11,15}$ This is important as several studies have found that pharmacy practice differs significantly between rural and urban areas. Scott et al. found that rural pharmacies offered more public health services compared to their urban counterparts. ${ }^{19}$ Others in the US found similar trends with pharmacy professional services. ${ }^{20,21}$ Guirguis et al. found in Canada that pharmacists in rural area were more likely to prescribe medications. ${ }^{26}$ While multiple definitions of rurality were used for these and other studies, it unknown whether the definitions impacted the results. Because healthcare policies and initiatives rely on data generated from healthcare research, inconsistency in the determination of geographic location may directly impact the creation and implementation of healthcare services in various areas. ${ }^{10,11,15}$

Healthcare services can also be indirectly impacted. Whether healthcare professionals, such as pharmacists, decide to work in an area may be influenced by their perception of the workplace's geographical setting. ${ }^{11}$ Pharmacists in particular play an integral role in the provision of healthcare due to their increased accessibility and prevalence; however, although nearly $20 \%$ of Americans live in rural areas, only $12 \%$ of pharmacists practice there. ${ }^{6,27,28}$ An awareness of their perception of rurality can therefore improve understanding of pharmacists location preferences and how this may impact rural communities. Thus, caution should be taken when relying on self-reported urban-rural categorizations to interpret healthcare behaviors and implement policies, as findings differ when based off zip-code derived rurality classifications.

There are several limitations to this study. Firstly, the original dataset was compiled to examine pharmacists' likelihood of providing hormonal contraception rather than to examine pharmacists' perception of rurality. Secondly, these data only looked at actively licensed pharmacists working in North Carolina and thus generalizability to other states may be limited. Further studies of other healthcare professionals in different areas are encouraged in order to establish a more encompassing study population. Thirdly, the response rate of the original study was low (5.9\%) and those who reported their zip code made up an even smaller percentage of those surveyed $(4.9 \%) .{ }^{22}$ As a result, the findings of this study may not accurately reflect the perceptions of all North Carolina pharmacists. Fourthly, we did not determine the impact of self-reported differences on pharmacy services availability. Finally, we did not examine why pharmacists self-reported a particular geographic setting and did not assess factors driving differences among those cases where there was conflict with the RUCA classification.

\section{CONCLUSIONS}

This study demonstrated some discordance between selfreported rurality and zip-code derived RUCA codes among pharmacists. Researchers and policy makers examining pharmacy services should be aware of this discordance and incorporate the use of standardized metrics, when possible. Future research should focus on the impact of self-reported geographic setting among pharmacists and healthcare professionals, particularly as it relates to the individual understandings of geographical location and healthcare accessibility within rural and urban communities.

\section{CONFLICT OF INTEREST}

The authors report no conflicts of interest with this research.

\section{FUNDING}

No external funding was provided for this work.

\section{AUTHOR ROLES (CRediT)}

Conceptualization: $\mathrm{CT}$.

Data curation: $\mathrm{CT}$.

Formal analysis: MC. Investigation: $\mathrm{MC}, \mathrm{CT}$.

Methodology: $\mathrm{CT}$.

Project administration: $\mathrm{MC}, \mathrm{CT}$.

Resources: MC, CT.

Software: MC.

Supervision: $\mathrm{CT}$.

Validation: $\mathrm{MC}$

Writing: original draft: $\mathrm{MC}, \mathrm{CT}$

Writing: review \& editing: $\mathrm{MC}, \mathrm{CT}$.

\section{References}

1. United States Census Bureau. 2010 Urban Area FAQs. https://www.census.gov/programssurveys/geography/about/faq/2010-urban-area-faq.html (accessed Oct 21, 2020). 
2. Kaczynski AT, Eberth JM, Stowe EW, et al. Development of a national childhood obesogenic environment index in the United States: differences by region and rurality. Int J Behav Nutr Phys Act. 2020;17(1):83. https://doi.org/10.1186/s12966-020-00984-x

3. Bennett K, Olatosi B, Probst J. Health disparities: Rural-urban chartbook. Columbia, SC: South Carolina Rural Health Research Center; 2008.

4. O'Connor A, Wellenius G. Rural-urban disparities in the prevalence of diabetes and coronary heart disease. Public Health. 2012;126(10):813-820. https://doi.org/10.1016/j.puhe.2012.05.029

5. Sanders SR, Cope MR, Park PN, Jeffery W, Jackson JE. Infants without health insurance: Racial/ethnic and rural/urban disparities in infant households' insurance coverage. PLoS One. 2020;15(1):e0222387. https://doi.org/10.1371/journal.pone.0222387

6. Talbert J, Schadler A, Freeman P. Rural/urban disparities in pneumococcal vaccine service delivery among the fee-forservice medicare population. Grand Forks: Rural \& Underserved Health Research Center; 2018.

7. Hart LG, Larson EH, Lishner DM. Rural definitions for health policy and research. Am J Public Health. 2005;95(7):11491155. https://doi.org/10.2105/ajph.2004.042432

8. Davis MM, Spurlock M, Dulacki K, et al. Disparities in Alcohol, Drug Use, and Mental Health Condition Prevalence and Access to Care in Rural, Isolated, and Reservation Areas: Findings From the South Dakota Health Survey. J Rural Health. 2016;32(3):287-302. https://doi.org/10.1111/jrh.12157

9. Fan JX, Wen M, Kowaleski-Jones L. Rural-urban differences in objective and subjective measures of physical activity: findings from the National Health and Nutrition Examination Survey (NHANES) 2003-2006. Prev Chronic Dis. 2014;11:E141. https://doi.org/10.5888/pcd11.140189

10. Martins SL, Starr KA, Hellerstedt WL, Gilliam ML. Differences in Family Planning Services by Rural-urban Geography: Survey of Title X-Supported Clinics In Great Plains and Midwestern States. Perspect Sex Reprod Health. 2016;48(1):9 16. https://doi.org/10.1363/48e7116

11. Lin GE, Rosenthal TC, Horwitz M. Physician location survey: self-reported and census-defined rural/urban locations. Soc Sci Med. 1997;44(11):1761-1766. https://doi.org/10.1016/s0277-9536(96)00378-4

12. Lor M, Bowers BJ, Krupp A, Jacobson N. Tailored Explanation: A Strategy to Minimize Nonresponse in Demographic Items Among Low-income Racial and Ethnic Minorities. Surv Pract. 2017;10(3):10.29115/SP-2017-0015. https://doi.org/10.29115/sp-2017-0015

13. Emmet M, Stein Q, Thorpe E, Campion M. Experiences of Genetic Counselors Practicing in Rural Areas. J Genet Couns. 2018;27(1):140-154. https://doi.org/10.1007/s10897-017-0131-6

14. Howarth HD, Peterson GM, Jackson SL. Does rural and urban community pharmacy practice differ? A narrative systematic review. Int J Pharm Pract. 2020;28(1):3-12. https://doi.org/10.1111/ijpp.12567

15. Cohen SA, Cook SK, Sando TA, Sabik NJ. What Aspects of Rural Life Contribute to Rural-Urban Health Disparities in Older Adults? Evidence From a National Survey. J Rural Health. 2018;34(3):293-303. https://doi.org/10.1111/jrh.12287

16. Onega T, Weiss JE, Alford-Teaster J, Goodrich M, Eliassen MS, Kim SJ. Concordance of Rural-Urban Self-identity and ZIP Code-Derived Rural-Urban Commuting Area (RUCA) Designation. J Rural Health. 2020;36(2):274-280. https://doi.org/10.1111/jrh.12364

17. Scott DM. Assessment of pharmacists' perception of patient care competence and need for training in rural and urban areas in North Dakota. J Rural Health. 2010;26(1):90-96. https://doi.org/10.1111//.1748-0361.2009.00270.x

18. Scott DM, Neary TJ, Thilliander T, Ueda CT. Factors affecting pharmacists' selection of rural or urban practice sites in Nebraska [published correction appears in Am J Hosp Pharm 1992 Oct;49(10):2444]. Am J Hosp Pharm. 1992;49(8):1941-1945.

19. Scott DM, Strand M, Undem T, Anderson G, Clarens A, Liu X. Assessment of pharmacists' delivery of public health services in rural and urban areas in lowa and North Dakota. Pharm Pract (Granada). 2016;14(4):836. https://doi.org/10.18549/pharmpract.2016.04.836

20. Haag JD, Stratton TP. Patient care services in rural Minnesota community pharmacies. J Am Pharm Assoc (2003) 2010;50(4):508-516. https://doi.org/10.1331/japha.2010.09134

21. Sisson EM, Israel MK. Extent of community-based delivery of pharmaceutical care in Virginia. Pharmacotherapy 1996;16(1):94-102. https://doi.org/10.1002/i.1875-9114.1996.tb02921.x

22. Seamon GJ, Burke A, Tak CR, Lenell A, Marciniak MW, Scott MA. Role of Pharmacists in Hormonal Contraceptive Access: A Survey of North Carolina Pharmacists. Pharmacy (Basel). 2020;8(4):191. https://doi.org/10.3390/pharmacy8040191

23. United States Department of Agriculture Economic Research Service. Rural-Urban Commuting Area Codes. https://www.ers.usda.gov/data-products/rural-urban-commuting-area-codes/ (accessed Oct 4, 2020).

24. WWAMI Rural Health Research Center. RUCA Data Using RUCA Data. https://depts.washington.edu/uwruca/rucauses.php (accessed Oct 23, 2020).

25. Health Resources and Services Administration. Defining Rural Population. https://www.hrsa.gov/rural-health/aboutus/definition/index.html (accessed Dec 1, 2020).

26. Guirguis LM, Hughes CA, Makowsky MJ, Sadowski CA, Schindel TJ, Yuksel N. Survey of pharmacist prescribing practices in Alberta. Am J Health Syst Pharm. 2017;74(2):62-69. https://doi.org/10.2146/ajhp150349

27. Poudel A, Lau ETL, Deldot M, Campbell C, Waite NM, Nissen LM. Pharmacist role in vaccination: Evidence and challenges. Vaccine. 2019;37(40):5939-5945. https://doi.org/10.1016/j.vaccine.2019.08.060

28. National Rural Health Association. Recruitment and retention of a quality health workforce in rural areas. Alexandria, VA; 2005. 